\title{
Early-onset epilepsy and microcephaly-capillary malformation syndrome caused by a novel STAMBP mutation in a Chinese boy
}

\author{
FANGRUI WU ${ }^{1-3 *}$, YING DAI $^{2-4^{*}}$, JUAN WANG $^{1-3}$, MIN CHENG $^{1-3}$, YANQIN WANG $^{1-3}$, \\ XIUJUAN LI $^{1-3}$, PING YUAN ${ }^{1-3}$, SHUANG LIAO ${ }^{1-3}$, LI JIANG $^{1-3}$, \\ JIN CHEN $^{1-3}$, LISI YAN ${ }^{1-3}$ and MIN ZHONG ${ }^{1-3,5}$ \\ ${ }^{1}$ Department of Neurology, Children's Hospital of Chongqing Medical University; ${ }^{2}$ Pediatric Research Institute, \\ China International Science and Technology Cooperation Base of Child Development and Critical Disorders, Chongqing \\ Engineering Research Center of Stem Cell Therapy, Ministry of Education Key Laboratory of Child Development and \\ Disorders; ${ }^{3}$ National Clinical Research Center for Child Health and Disorders; ${ }^{4}$ Department of \\ Primary Child Health Care, Children's Hospital of Chongqing Medical University, Chongqing 400014; \\ ${ }^{5}$ Department of Pediatric, Qianjiang Central Hospital of Chongqing, Chongqing 409000, P.R. China
}

Received May 19, 2019; Accepted September 9, 2019

DOI: $10.3892 / \mathrm{mmr} .2019 .10757$

\begin{abstract}
To the best of our knowledge, the present study reported the case of the first Chinese patient with microcephaly-capillary malformation (MIC-CAP) syndrome caused by a novel compound heterozygous mutation in the STAMBP gene, which encodes STAM binding protein. The present study also provides a review of relevant previously published studies. A boy with MIC-CAP syndrome with developmental delay, intractable epilepsy and prominent dyskinesia was examined. A pathogenic mutation was identified by whole-exome sequencing, and the protein structure and function affected by this mutation were predicted using bioinformatics analysis. Finally, the clinical features of 16 other cases reported in previous studies were reviewed and compared. A novel compound heterozygous mutation of the STAMBP (c.1119-1G>T, c.968A $>$ G) was identified in the present study and epilepsy was refractory, consistent with previously reported cases. The present study also highlighted the fact that STAMBP mutation-associated MIC-CAP often presents as intractable early-life epilepsy, which may lead to mortality.
\end{abstract}

Correspondence to: Dr Min Zhong, Department of Neurology, Children's Hospital of Chongqing Medical University, 136 Zhongshan 2nd Road, Chongqing 400014, P.R. China

E-mail: zhongmin@hospital.cqmu.edu.cn

${ }^{*}$ Contributed equally

Key words: epilepsy, microcephaly, mutation, spasm, STAM binding protein

\section{Introduction}

Mutations in the STAM binding protein (STAMBP) gene cause microcephaly-capillary malformation (MIC-CAP) syndrome (OMIM, 614261; ORPHA, 294016), which was described as a novel syndrome several years ago $(1,2)$. Elevated ubiquitin-conjugated protein aggregation and apoptosis activation has previously been identified in lymphoblastoid cell lines from patients with MIC-CAP caused by STAMBP mutations. Additionally, elevated autophagosome content, active and insensitive RAS-MAPK and PI3K-AKT-mTOR pathways have also been found in this syndrome (1).The MIC-CAP syndrome is a rare neurocutaneous disorder characterized by congenital microcephaly, early-onset epilepsy, severe profound developmental delay and diffuse cutaneous capillary malformations (1-3). In total, 18 pathogenic mutations have been reported in 16 patients from 8 ethnic groups (1-8).

To the best of our knowledge, the present study reported the case of the first Chinese patient with MIC-CAP syndrome caused by a novel compound heterozygous STAMBP mutation. The present study also provided a review of relevant previously published cases.

\section{Patients and methods}

Patients. Ethical approval for the present study was obtained from the Institutional Review Board, Children's Hospital of Chongqing Medical University (permit no. 2018-64). Informed consent was obtained from the parents of the patient.

The case described in the present study is that of a boy born after 40 weeks and 5 days of gestation, G2P1 (second pregnancy and first successful birth of the mother), to nonconsanguineous Chinese parents (father, 23 years old; mother, 22 years old). The boy was born through normal 
delivery, with a birth weight of 3,600 g. The birth length and head circumference were not known.

The patient was admitted (June 2018) to the Department of Neurology, Children's Hospital of Chongqing Medical University at the age of 1 year and 5 months. A routine examination of general health and neurological evaluations were carried out. Magnetic resonance imaging (MRI), electroencephalography and metabolic screening were performed according to the manufacturer's protocols. All the available clinical characteristics of the patient along with the aforementioned auxiliary examination results are summarized in the present study.

Genetic analysis. Peripheral blood samples $(5 \mathrm{ml})$ from the proband and the parents were collected into graded negative pressure vacuum EDTA anticoagulant tubes. All sample preparation, whole-exome sequencing and Sanger sequencing were performed by Beijing Mygenostics Co, Ltd. Several online databases containing data from different ethnic groups were used as following; dbSNP (http://www.ncbi.nlm.nih.gov/projects/SNP/),ExAC (http://exac.broadinstitute.org/faq), Genome Aggregation Database (gnomAD, http://gnomad.broadinstitute.org/), Clinvar (https://www.ncbi.nlm.nih.gov/clinvar/), esp6500siv2_all (http://evs.gs.washington.edu/EVS/). 1000 genome (http://www.1000genomes.org), Mutation screening of $S T A M B P$ was performed using Sanger sequencing with two primer pairs to amplify exon 9 (chr2-74087178) and exon 7 (chr2-74077603) of the STAMBP gene (NM_201647). The following primers were used: Exon 9, forward 5'-CTCACA ATGACCTCTGCCCT-3', reverse 5'ATTCCTGTCCCACAC TGCTT-3'; and exon 7, forward 5'GAGCACCAGGGAATT GTGAC-3' and reverse 5'AAGCCCTAAGTGTTCCCAGA-3'. BigDye Terminator v3.1 Cycle Sequencing kit (Invitrogen; Thermo Fisher Scientific, Inc.) was used and thermal cycling was performed in a Mix Golden Star T6 Super PCR Mix (1.1x; TsingKe Biological Technology Co.; Table I). The segregation of the identified mutations was investigated in the parents.

The prediction of mutations was assessed using software, including PolyPhen_2_Predict, PolyPhen_2 (http://genetics. bwh.harvard.edu/pph2/), SIFT, SIFT_Predict (http://sift. jcvi.org/), SPIDEX (http://www.deepgenomics.com/spidex), MutationTaster, MutationTaster_Predict (http://www.mutationtaster.org/ChrPos.html), MCAP_score, MCAP_pred (http://bejerano.stanford.edu/MCAP/), GERP++_Predict, GERP++ (http://mendel.stanford.edu/SidowLab/downloads/gerp/index.html) and REVEL_score (https://sites.google. com/site/revelgenomics/). The protein structure of $S T A M B P$ was modelled using the SWISS-MODEL (3rzv.1, https://swissmodel.expasy.org/interactive)

Literature review. The PubMed (https://www.ncbi.nlm.nih. gov/pubmed/) and Wanfang (http://www.wanfangdata.com. $\mathrm{cn} /$ index.html) databases were used to retrieve previous studies using the keywords 'STAMBP, microcephaly, and capillary malformation' until April 2019. The publication language was limited to English and Chinese.

\section{Results}

Patient treatment. The child developed early-onset epilepsy after 3 months, with a generalized tonic-clonic seizure, which
Table I. Thermocycling conditions for Sanger sequencing.

\begin{tabular}{lccc}
\hline Step & Temperature $\left({ }^{\circ} \mathrm{C}\right)$ & Time & Cycle \\
\hline 1 & 95 & $10 \mathrm{~min}$ & 1 \\
2 & 94 & $30 \mathrm{sec}$ & 3 \\
& 64 & $30 \mathrm{sec}$ & \\
3 & 72 & $45 \mathrm{sec}$ & \\
& 94 & $30 \mathrm{sec}$ & 5 \\
& 62 & $30 \mathrm{sec}$ & \\
4 & 72 & $45 \mathrm{sec}$ & 10 \\
& 94 & $30 \mathrm{sec}$ & \\
5 & 60 & $30 \mathrm{sec}$ & 17 \\
& 72 & $45 \mathrm{sec}$ & \\
7 & 94 & $30 \mathrm{sec}$ & 1 \\
& 58 & $30 \mathrm{sec}$ & \\
\hline
\end{tabular}

progressed to clusters of infantile spasms (2-10 clusters/day) 1 month later. The patient was treated successively with levetiracetam (40-50 mg/kg/day), topiramate (6-7 mg/ $\mathrm{kg} /$ day), valproic acid (30 mg/kg/day) and corticosteroids at the outpatient clinic. The spasms decreased and became myoclonic, but the epilepsy remained refractory.

The patient was admitted to the neurologic ward after 1 year and 2 months. The patient had drooping mouth corners, a short nose and neck, and sporadic, multiple, small capillary malformations (Fig. 1A and B). Referring to the World Health Organization Anthro standards (https://www.who. $\mathrm{int} /$ childgrowth/software/en/), the patient was $8 \mathrm{~kg}$ [Z-score, -2.8 Standard Deviation (SD)] in weight and $63 \mathrm{~cm}$ (Z-score, $-6.8 \mathrm{SD})$ in length. The head circumference was $39.5 \mathrm{~cm}$ (Z-score, -5.8 SD). The boy could not hold his head and had prominent dyskinesia of the whole body, particularly involuntary movement of the tongue and mouth (Video S1).

The patient was on a ketogenic diet during the inpatient stay and received immunoglobulin intravenously as a result of recurrent pneumonia, 22 days later, he was discharged. During the follow-up, the seizures were still not well controlled, although the ketogenic diet ratio was modified from 2:1 to 4:1 ( $4 \mathrm{~g}$ fat/l $\mathrm{g}$ combined protein, carbohydrate). After 1 month, the patient received vigabatrin $(60 \mathrm{mg} / \mathrm{kg} /$ day $)$ and the seizures reduced by $80 \%$ a week later. Unfortunately, the patient suddenly succumbed 3 weeks later; no definitive causes were found as no autopsy was performed.

The MRI scans showed slightly dilated lateral ventricles and increased extra-axial spaces. Interictal electroencephalography showed hypsarrhythmia and slow wave background with bioccipital spike-slow wave during waking (Fig. 1C and D). Blood and urinary metabolic screening indicated normal results.

Exome sequencing. Exome sequencing revealed intronic (c.1119-1G>T) and exonic mutations in the proband and their parents, which was confirmed via Sanger sequencing (Fig. 2A). 


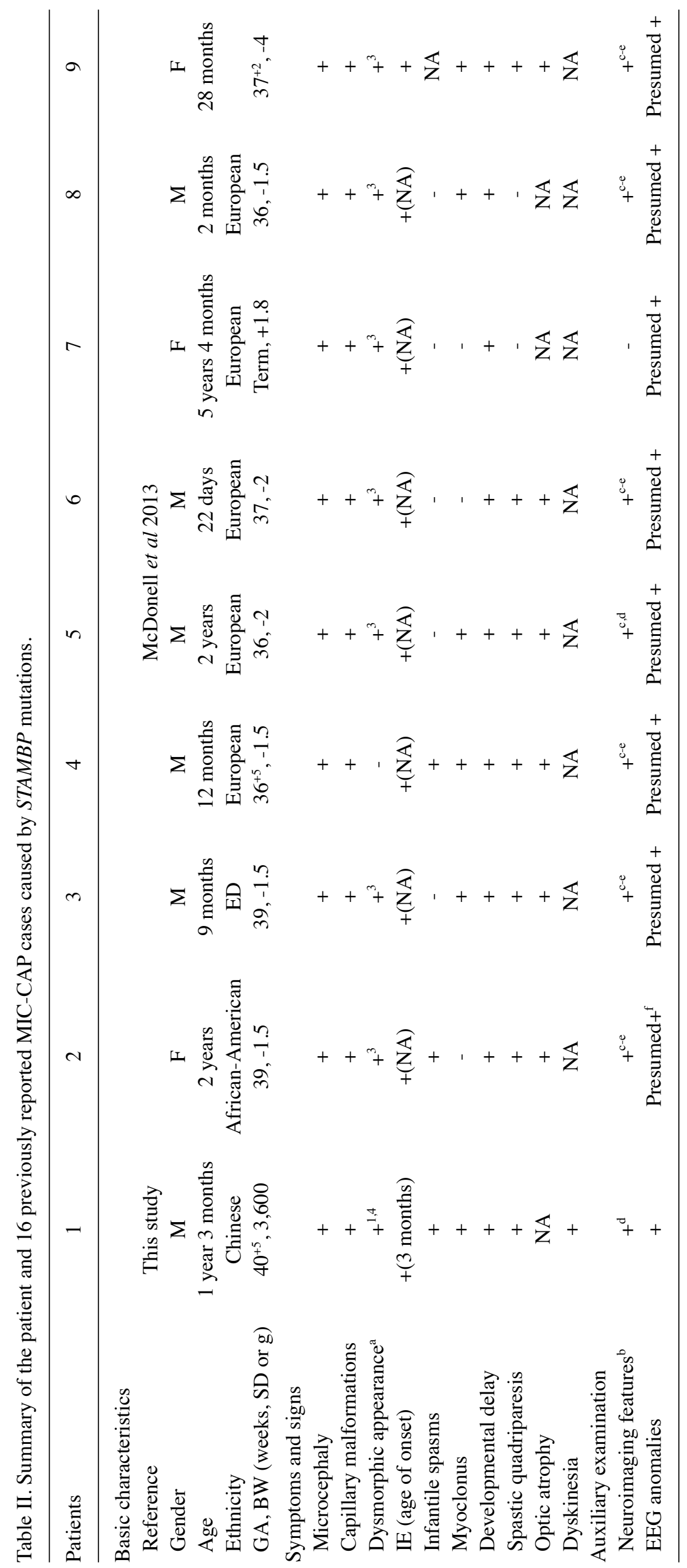




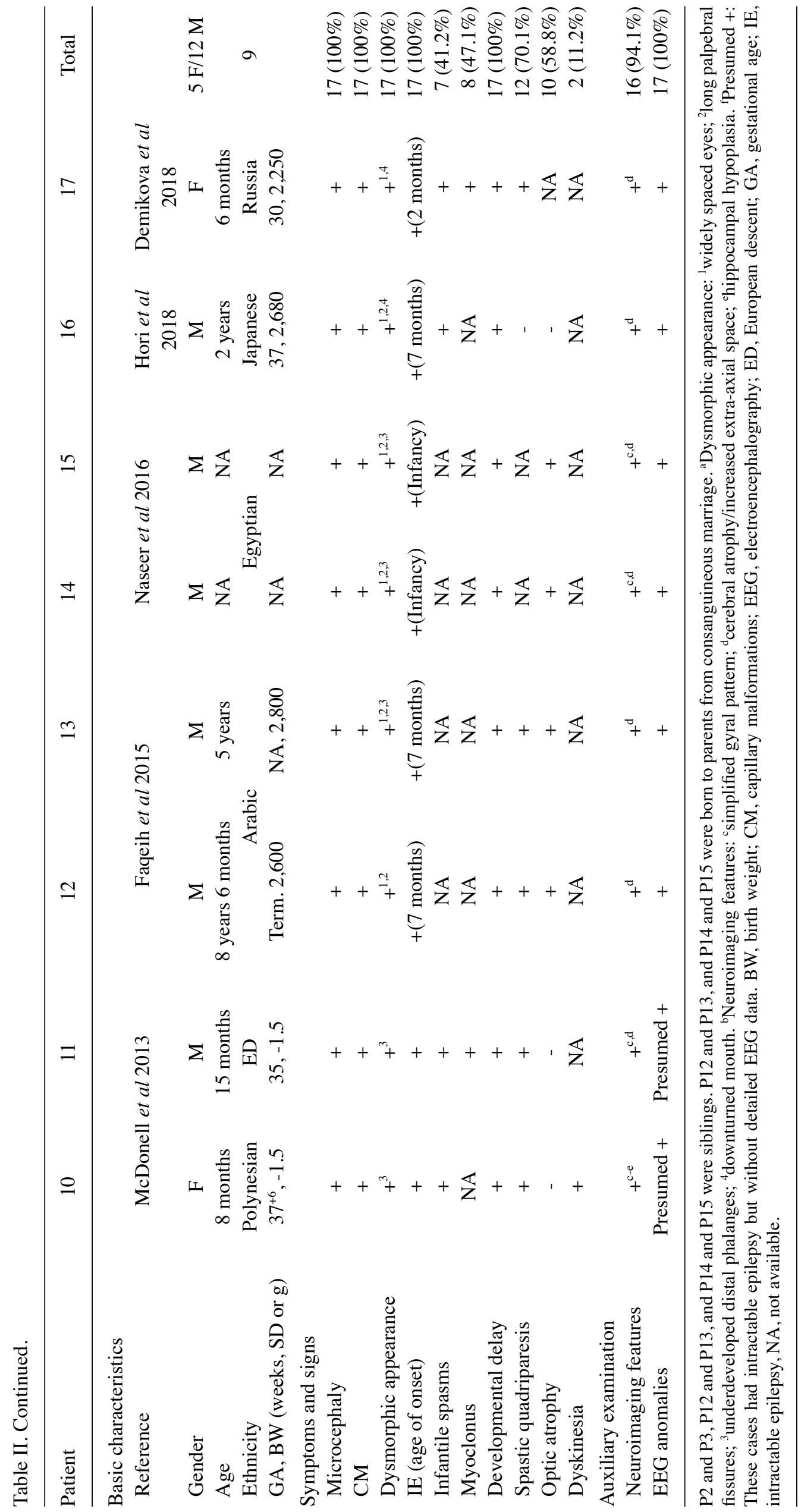


A

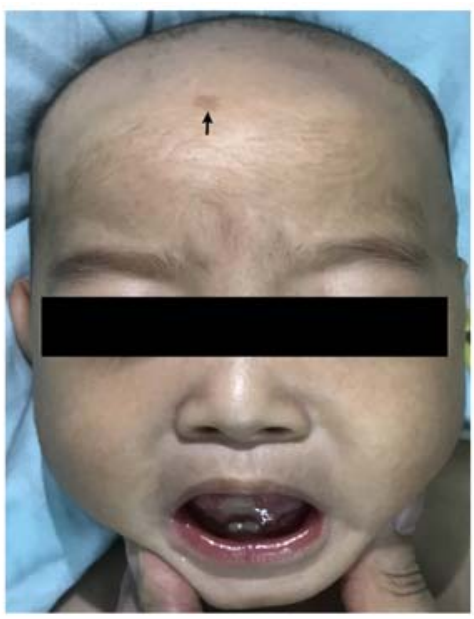

B

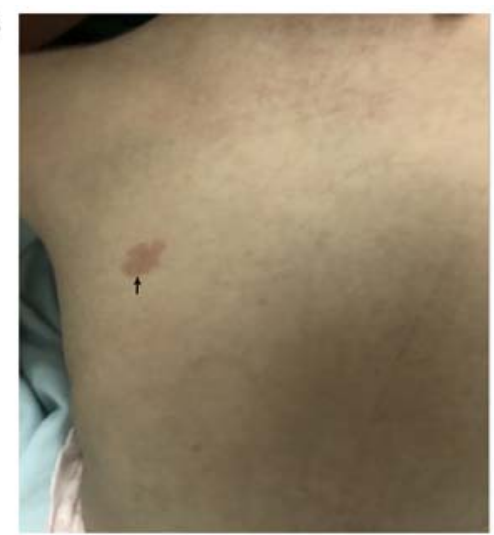

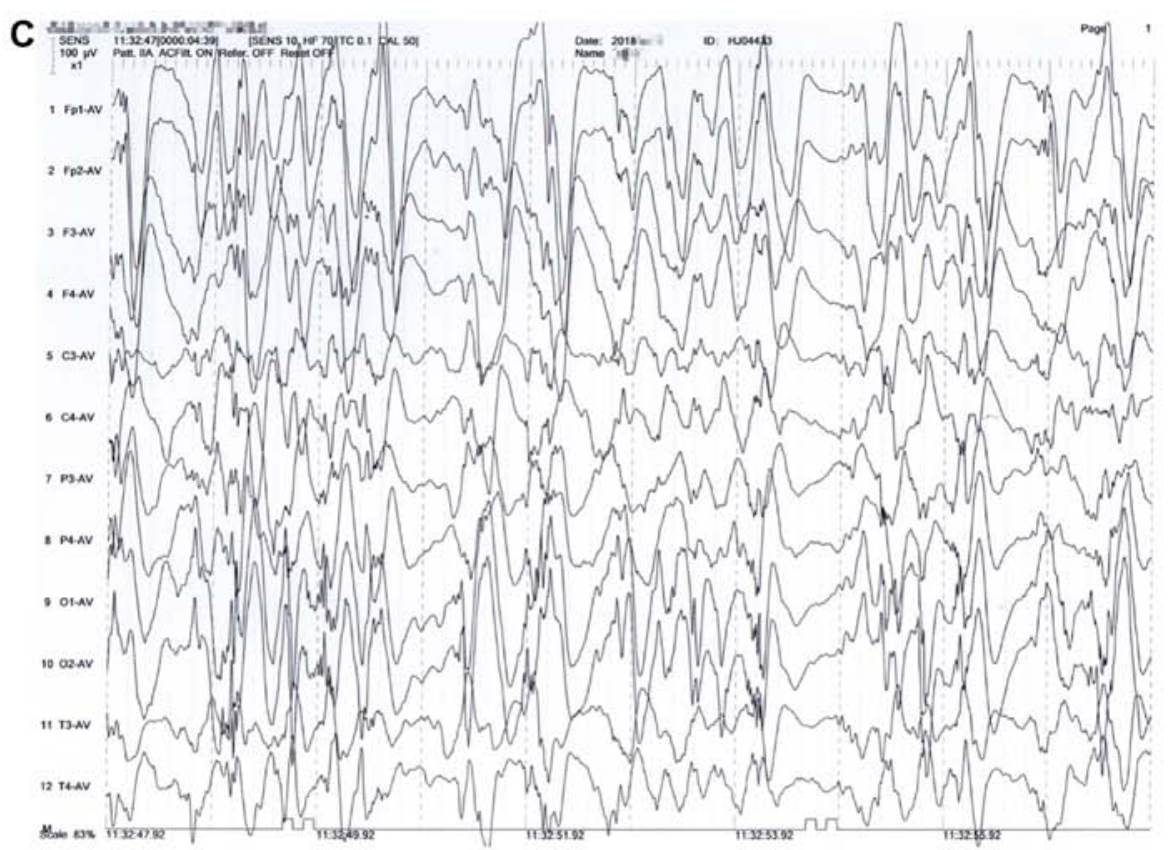

D

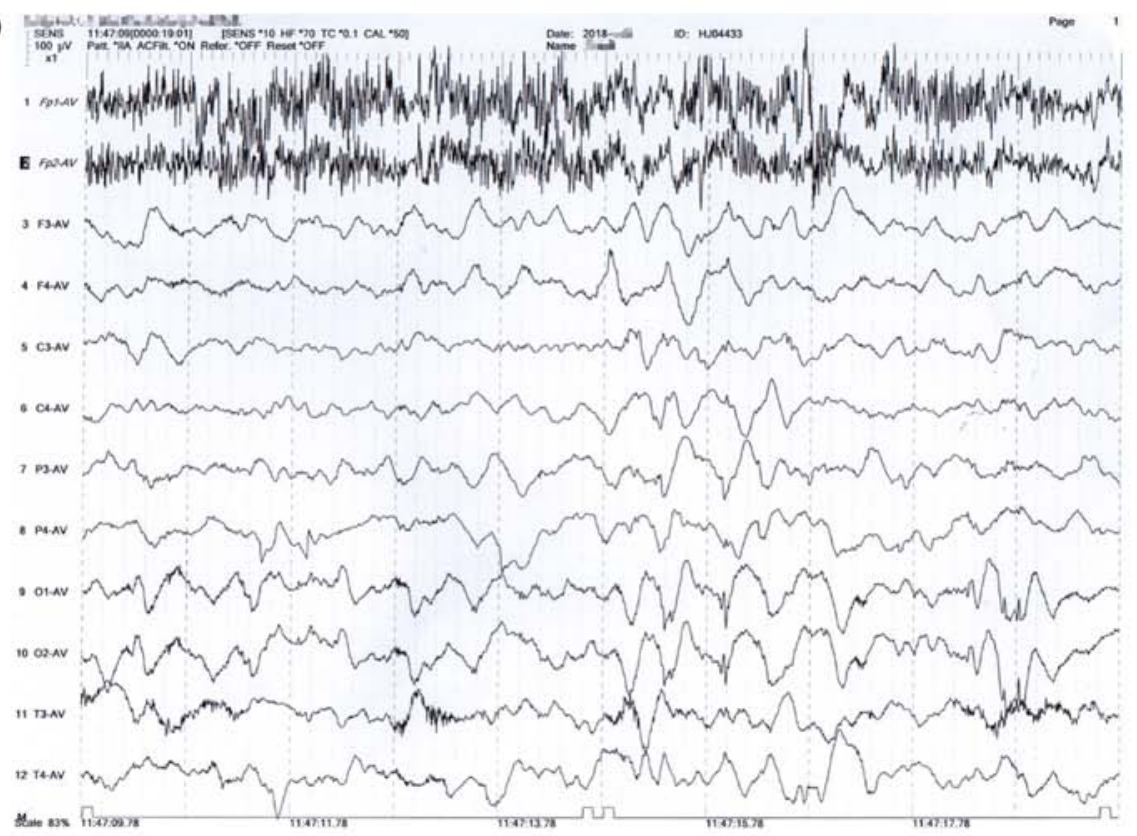

Figure 1. Case presentation. Sporadic multiple small capillary malformations (black arrows) found on the (A) forehead and (B) upper back. At the age of 12 months, interictal electroencephalogram revealed hypsarrhythmia $(C)$, and slow wave background with bioccipital spike slow wave (D) during waking. 


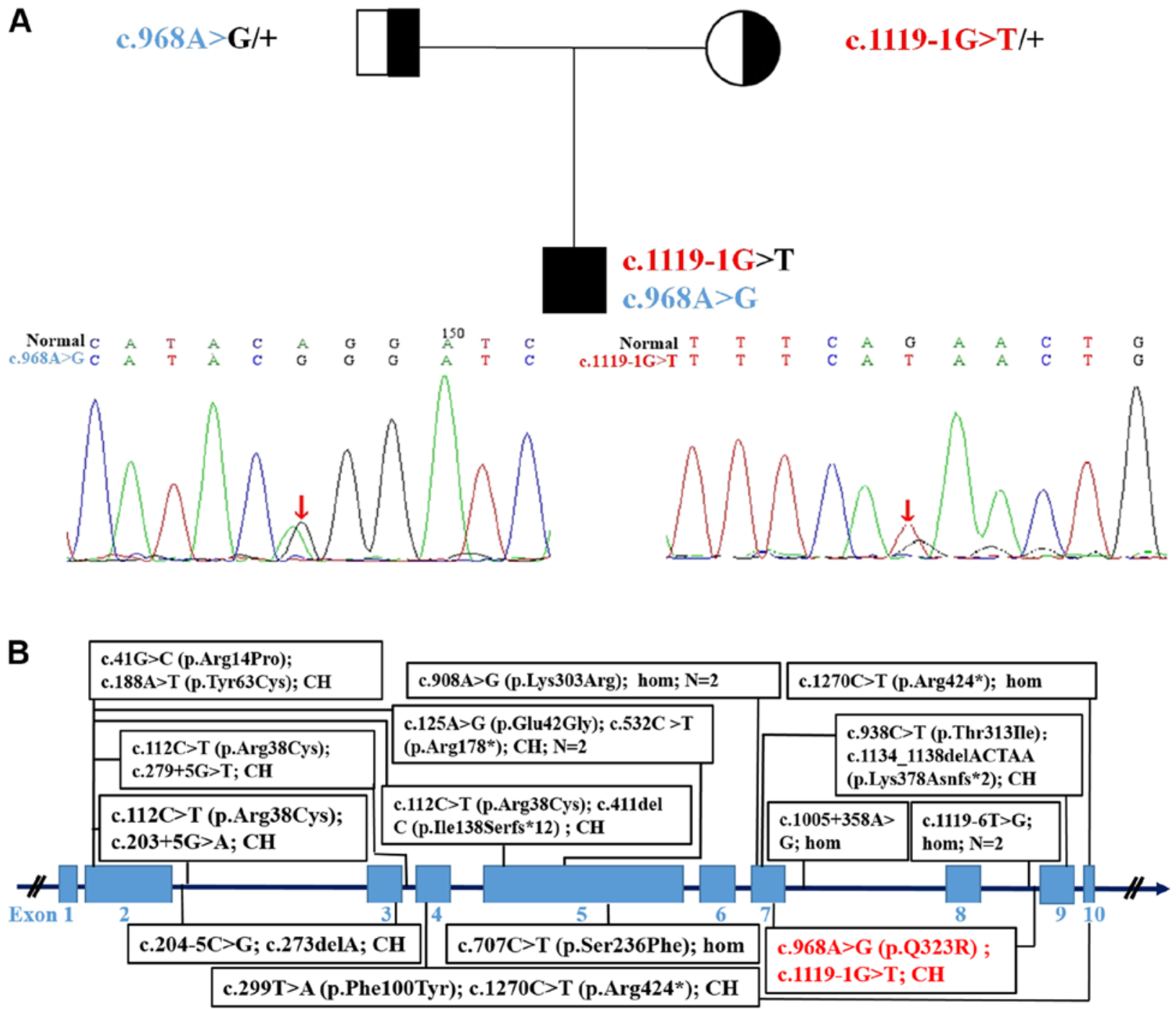

Figure 2. Pedigreeof the family. (A) Sanger sequencing showing a novel STAMBP compound heterozygosity mutation inherited from the parents. (B) Gene structure of $S T A M B P$, and its pathogenic variants in patients with microcephaly-capillary malformation reported previously and described in the present study (red). $\mathrm{CH}$, compound heterozygous; STAMBP, encodes STAM binding protein.

The mutations segregated according to a strictly recessive model with full penetrance. The parents were found to be heterozygous carriers, (mother, c.1119-1G $>\mathrm{T}$; father, c.968A $>$ G; Fig. 2A). The protein structures of wild-type and mutated STAMBP were modelled and predicted, respectively, as shown in Fig. S1. The mutations affected the amino acid side chain (p.Gln323Arg) and were predicted to be pathogenic. The splice site variant (c.1119-1G $>$ T) was predicted to generate aberrant splicing of the $S T A M B P$ mRNA.

The clinical features and pathogenic variants of 16 previously reported cases of MIC-CAP are summarized in Table II and Fig. 2B.

\section{Discussion}

So far as we know, the present study presented the first case of a Chinese patient affected by refractory epilepsy, microcephaly, severe developmental delay and diffuse cutaneous capillary malformations. These four symptoms were also observed in all of the patients studied in previous reports (100\%) (1-8). The patient in the present study also had spastic quadriparesis, without optic atrophy; however, both optic atrophy and spastic quadriparesis were common in previous cases (1-8). Moreover, dyskinesia was more prominent in the present study and was infrequent in previous cases, except in one patient (1). Although the patient in the present study responded well to vigabatrin, the long-term efficacy of the drug could not be evaluated due to the early death of the patient.

A novel compound heterozygous co-segregating mutation in STAMBP [c.1119-1G>T (splicing, exon9, maternal allele) and c.968A > G (p.Gln323Arg, exon7, paternal allele); Fig. 2A] was identified through exome sequencing. The parents of the patient were healthy. The variant frequency of the aforementioned mutation has not been reported in the Chinese Reference Genome Database or any public sequence database, to the best of our knowledge. In silico analysis indicated that this splice site mutation generated aberrant splicing of the STAMBP mRNA, and c.968A $>$ G was also predicted to be pathogenic. Therefore, a definite diagnosis for this patient with MIC-CAP could be made due to the autosomal recessive inheritance patterns, according to the previous reports (1-8). 
To date, only 16 cases (11 male and 5 female) from 13 families in 8 ethnic groups have been reported, with 18 different STAMBP mutations (1-8). All the parents were from unrelated families, except for two consanguineous relationships (4-6). A further two patients had causative mutations (c.753_754insT, c.1119-1G>T) $(6,8)$, which have been omitted from the figure as further details of these patients could not be found. In total, 20 STAMBP mutations are listed online without further patient details; therefore, their pathogenicity could not be confirmed (ClinVar).

In conclusion, to the best of our knowledge, the present study reported the first case of a Chinese patient with refractory epilepsy as an initial symptom of MIC-CAP. Additionally, novel pathogenic compound heterozygosity of the $S T A M B P$ was identified. The results of the present study may improve understanding of STAMBP mutations and ethnic background in cases MIC-CAP.

\section{Acknowledgements}

Not applicable.

\section{Funding}

The present study was supported in part by a grant from the Children's Hospital of Chongqing Medical University for Special Project on Difficult and Rare Diseases (grant no. HJYN2013-4).

\section{Availability of data and materials}

The datasets used and/or analyzed during the present study are available from the corresponding author on reasonable request.

\section{Authors' contributions}

FW, YD, YW and MZ conceived and designed the study, and analyzed the data; JW, MC, XL, PY, SL and LJ collected the clinical information; JC and LY performed the EEG analysis; FW, YD and MZ prepared the manuscript. All authors read and approved the final manuscript.

\section{Ethics approval and consent to participate}

Ethical approval for the present study was obtained from the Institutional Review Board, Children's Hospital of Chongqing Medical University (grant no. 2018-64).

\section{Patient consent for publication}

Informed consent was obtained from the parents of the patient.

\section{Competing interests}

The authors declare that they have no competing interests.

\section{References}

1. McDonell LM, Mirzaa GM, Alcantara D, Schwartzentruber J, Carter MT, Lee LJ, Clericuzio CL, Graham JM Jr, Morris-Rosendahl DJ, Polster T, et al: Mutations in STAMBP, encoding a deubiquitinating enzyme, cause microcephaly-capillary malformation syndrome. Nat Genet 45: 556-562, 2013.

2. Carter MT and Boycott KM: Microcephaly-capillary malformation syndrome: A story of rapid emergence of a new recognizable entity. Am J Med Genet A 155A: 2078-2079, 2011.

3. Carter MT, Geraghty MT, De La Cruz L, Reichard RR, Boccuto L, Schwartz CE and Clericuzio CL: A new syndrome with multiple capillary malformations, intractable seizures, and brain and limb anomalies. Am J Med Genet A 155A: 301-306, 2011.

4. Pavlovic M, Neubauer D, Al Tawari A and Heberle LC: The microcephaly-capillary malformation syndrome in two brothers with novel clinical features. Pediatr. Neurol 51: 560-565, 2014

5. Faqeih EA, Bastaki L, Rosti RO, Spencer EG, Zada AP, Saleh MA, Um K and Gleeson JG: Novel STAMBP mutation and additional findings in an Arabic family. Am J Med Genet A 167: 805-809, 2015.

6. Naseer MI, Sogaty S, Rasool M, Chaudhary AG, Abutalib YA, Walker S, Marshall CR, Merico D, Carter MT, Scherer SW, et al: Microcephaly-capillary malformation syndrome: Brothers with a homozygous STAMBP mutation, uncovered by exome sequencing. Am J Med Genet A 170: 3018-3022, 2016.

7. Demikova NS, Kakaulina VS, Pechatnikova NL, Polyakova NA Zakharova EY, Krylova TD and Zubkova MV: First report of microcephaly-capillary malformations syndrome in Russia. Egypt J Med Hum Genet 19: 147-150, 2018.

8. Hori I, Miya F, Negishi Y, Hattori A, Ando N, Boroevich KA, Okamoto N, Kato M, Tsunoda T, Yamasaki M, et al: A novel homozygous missense mutation in the SH3-binding motif of STAMBP causing microcephaly-capillary malformation syndrome. J Hum Genet 63: 957-963, 2018.

This work is licensed under a Creative Commons Attribution-NonCommercial-NoDerivatives 4.0 International (CC BY-NC-ND 4.0) License. 